\title{
Evaluating the analgesic effect of the GLS inhibitor 6-diazo-5-oxo-I-norleucine in vivo
}

\begin{abstract}
Glutamate is an excitatory neurotransmitter, produced by its synthetic enzyme, glutaminase (GLS), and packaged by vesicular transporters (VGluT2) into synaptic vesicles. Primary sensory peripheral nerve and spinal synaptic terminals release glutamate during nociceptive (pain) signaling. In post-incisional and inflammation models in rats, GLS and VGluT2 production is elevated in dorsal root ganglion neuronal cell bodies and transported to peripheral and spinal terminals for increased glutamate synthesis and release. 6-Diazo-5-oxo-1-norleucine (DON) is a GLS inhibitor that produces long lasting pain relief when applied to the inflamed paw of arthritic rats, but its effect in a post-incisional model has not been evaluated. In this study, we examined the analgesic efficacy of DON in a surgical incision model by measuring thermal latency and mechanical allodynia. Following behavioral evaluation, we examined the skin for VGluT2, GLS and glutamate immunoreactivity (ir). Our findings revealed that VGluT2-ir is elevated in the stratum lucidum by approximately $19 \%$, 64hours post-surgical incision and attenuated by approximately $5.4 \%$ after the administration of DON. During that same period GLS-ir was elevated in dermal nerve fibers by $52 \%$ and was attenuated by approximately $27.9 \%$ after the application of DON. Additionally, glutamate-ir was elevated in epidermal nerve fibers by $35 \%$ after incision and attenuated by approximately $23 \%$ after the administration of DON. Behavioral testing 24 and 48hours after a single local administration of DON showed five out of six animals having an analgesic response to mechanical allodynia, but not to thermal hyperalgesia. Following a surgical incision, the area of injury shows increased VGluT2-, GLS-, glutamate-ir, mechanical allodynia and no change in thermal latency. After the application of the GLS inhibitor, DON, nerve fiber of the skin showed decreased VGluT2, GLS, and glutamate-ir. Furthermore, post-incision DON treated animals exhibited decreased mechanical allodynia with no change in thermal latency when compared to control animals.
\end{abstract}

Keywords: glutaminase, glutamate, incision, post-surgical pain, vglut2, DRG, sprague-dawley rats, GLS, don, 6-diazo-5-oxo-1-norleucine
Volume 3 Issue 3 - 2016

\author{
Heith A Crosby, Kenneth E Miller \\ Department of Anatomy and Cell Biology, Oklahoma State \\ University, USA
}

\begin{abstract}
Correspondence: Heith A Crosby, Department of Anatomy and Cell Biology, Oklahoma State University -Center for Health Sciences, IIII West 17th Street Tulsa, Oklahoma 74107, USA, Tel 9185615817, Email heith.crosby@okstate.edu
\end{abstract}

Received: October 23, 2015 | Published: January 08, 2016

\begin{abstract}
Abbreviations: AIA, adjuvant-induced arthritis; AST, aspartate amino transferase; BSA, bovine serum albumin; DON, 6-diazo-5oxo-1-norleucine; DRG, dorsal root ganglia; GLU, glutamate; GLS, glutaminase; IR, immunoreactivity; MRNA, messenger rna; NSAID, non-steroidal anti-inflammatory drug; PBS, phosphate buffered saline; PBST, phosphate buffered saline with triton $\mathrm{x}-100$; PPP, post-surgical pain; PVP, polyvinylpyrollidone; VGLUT2, vesicular glutamate transporter 2
\end{abstract}

\section{Introduction}

Surgical procedures are common in the United States with some estimates greater than 99 million per year. A serious clinical problem that can occur because of surgery is the condition of persistent postsurgical pain (PPP). This has been defined as pain that lasts longer than three months after surgery. The incidence of PPP has been reported to be as high as $40 \%$, with greater than $18 \%$ of patients reporting their pain as moderate to severe. ${ }^{1}$ The physiological consequences of inadequately controlled post-operative pain can result in various complications (i.e., respiratory, cardiovascular, thromboembolic, gastrointestinal, musculoskeletal, and psychological), all of which can delay or impair patient recovery and increase the total cost of care. Inadequate post-operative pain control may also lead to the development of chronic pain after surgery. These problems highlight the overarching goal of post-operative pain management, which is to avoid post-operative complications and the development of chronic pain, thereby improving the comfort of the patient, facilitating recovery, reducing morbidity and promoting quicker discharge from the hospital. ${ }^{2}$

\section{Pre-emptive analgesia}

Pre-emptive or preventive analgesia is defined "as the administration of analgesia before surgical incision to prevent establishment of central sensitization from incision or inflammatory injury in order to achieve optimal post-operative pain control." The results of clinical trials on the efficacy of pre-emptive analgesia are controversial due to the difficulty in differences in noxious stimuli intensity, verification of the direct pharmacological effect of treatment, differences in drug concentrations between study groups, and outcome measurements. ${ }^{3}$

\section{Glutaminase in primary sensory neurons}

Glutamate (GLU) is the amino acid neurotransmitter used by primary sensory neurons of the dorsal root ganglia (DRG). This excitatory neurotransmitter is released from the peripheral nerve and spinal synaptic terminals during nociceptive (pain) signaling. ${ }^{4}$ GLU is produced either by cytosolic transamination of aspartate by aspartate aminotransferase (AST) or via the hydrolytic deamination 
of glutamine by the mitochondrial metabolic enzyme, glutaminase (GLS). In neurons, GLS is the primary producer of glutamate. ${ }^{5}$

GLS is elevated in both the cytoplasm and mitochondria of primary afferent sensory neuronal cell bodies in the adjuvant-induced arthritis (AIA) and surgical incision models in the rat. ${ }^{6-8}$ GLS protein expression, enzyme activity, and immunoreactivity (ir) are elevated in DRG neuronal cell bodies from 1-8days of AIA. The elevation of GLS-ir is most prominent in nociceptive ' $\mathrm{C}$ ' type neurons at 8-12days of AIA. Following increased production in the cell soma during AIA, GLS is transported in peripheral axons causing increased GLU synthesis in peripheral nerve terminals. ${ }^{9}$ Furthermore, we have demonstrated that both GLS and VGluT2-ir are elevated post-surgical incision in $\mathrm{S}_{1}$ DRG neurons for up to 72hours, while GLS mRNA levels rapidly decrease post-incision and remain depressed for at least 96hours. ${ }^{8}$

Since GLS is responsible for the neuronal production of glutamate from glutamine, it is a potential therapeutic target for alleviating pain. $7,10,11$ The GLS enzyme contains several regulatory sites ${ }^{12,13}$ and several classes of GLS inhibitors, acting at both allosteric and enzymatic sites are effective analgesics when injected into the inflamed limb of adjuvant-induced arthritis (AIA) animals. ${ }^{7}$ Miller et al. ${ }^{7}$ examined if inhibition of GLS in peripheral terminals would reduce elevated glutamate levels and provide pain relief during chronic inflammation. After a single intra-plantar injection of DON in rats with 3days AIA, thermal and mechanical hyperalgesia responses were comparable to control animals and glutamate-ir appeared similar to controls. ${ }^{7}$ Other experiments have shown that pre-treatment of rat hind paws with DON, along with co-administration of DON at the start of inflammation with carrageenan, decreased paw swelling by $10 \%$ and reduced the number of activated neurons in the spinal cord as demonstrated by c-fos-ir. ${ }^{10}$

Based on these findings and our discovery that DRG neurons increase GLS and VGluT2 following surgical incision similar to AIA, we investigated if a topical application of DON into a surgical incision will have a similar effect compared to the AIA model. We analyzed tail thermal and mechanical responses and evaluated skin ir for the glutamatergic biomarkers, VGluT2, GLS and GLU.

\section{Materials and methods}

\section{Animals}

Harlan Sprague-Dawley rats (Oklahoma State University Center for Health Sciences breeding colony originating from Charles River) were housed on a 12 hour light: 12 hour dark cycle and given free access to food and water. Procedures were conducted according to guidelines from the National Institutes of Health ${ }^{11}$ and were approved by the Oklahoma State University Center for Health Sciences Institutional Animal Care and Use Committee. All appropriate efforts were made to minimize the number of animals used in these studies.

\section{Surgical incision}

Harlan Sprague-Dawley rats $(n=21$, male and female), weighing between 250 -350grams (mean weight $=300.2$ grams, mean age $=252.4$ days), were anesthetized with $5 \%$ isoflurane and oxygen ( 3 $\mathrm{L} / \mathrm{min}$ ) in a plastic induction chamber until mobility ceased. A nose cone was utilized to deliver maintenance anesthesia (isoflurane 2-3\%, $\mathrm{O}_{2} 1.5 \mathrm{~L} / \mathrm{min}$ ). The tails of the rats were cleaned using povidone iodine for disinfection of the surgical site. The experimental animals $(n=15)$ had a $20 \mathrm{~mm}$ midline incision made through skin, fascia and muscle on the dorsal surface of the tail. The incisions were made longitudinally, along the midpoint of the proximal third of the tail, with half the incision above and half below the proximal third midpoint. The incisions were sutured using a $19 \mathrm{~mm}$ reverse cutting needle with two 5.0 silk sutures. Control animals $(n=6)$ were surgical naïve rats that underwent anesthesia and disinfection as described previously.

\section{DON administration}

Animals were anesthetized as described previously. Using a tuberculin syringe fitted with a 27 -gauge needle, twenty $0.05 \mathrm{ml}$ aliquots of DON (2mM) were infiltrated into the wound margins bilaterally and down the midline of the incision. For control animals that received DON, similar injections were given by intradermal administration.

\section{Experimental time line}

All animals were acclimatized to behavior testing (e.g., thermal and mechanical) daily for 3 days prior to the initiation of the experiment. On Day 0, baseline tests were performed for both thermal and mechanical responses and data recorded. Behavior tests were performed and data recorded at $0,16,40$ and 64hours after the initiation of the experiment. Animals that received surgical incisions (i.e., incision only and incision with DON groups) had behavioral testing after the surgical incision procedure. Injections were given 16hours after the incision time point (0hour) for both groups that received DON injections (i.e., DON injection only and incision with DON). The 16hour time point post surgical incision was chosen to allow for newly produced GLS enzyme as a result of the surgical incision adequate time to travel from associated DRG to their peripheral terminals. By timing the injection with the rise in GLS enzyme at the peripheral terminals we would best demonstrate DON's mechanism of action (i.e., GLS inhibition). For control animals, only behavior acclimation and testing were performed. Following the final behavior testing, all animals underwent transcardial perfusions and skin tissue was collected from the proximal portion of the tail (Figure $1)$.

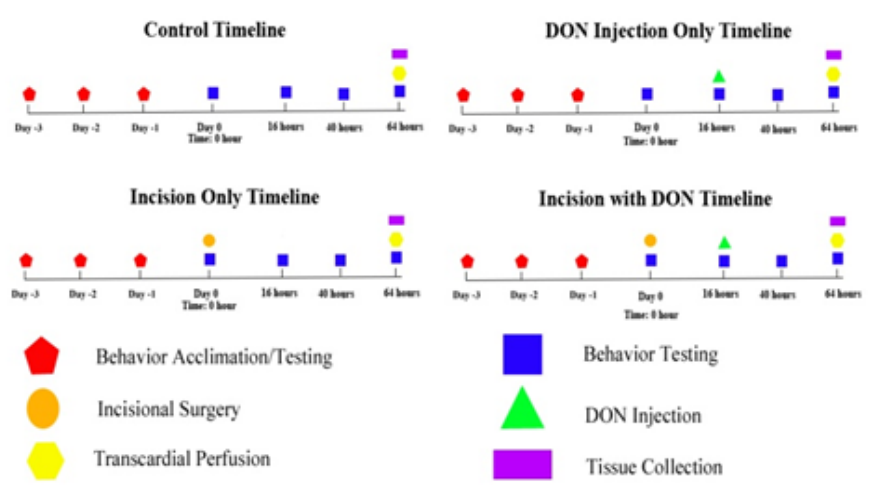

Figure I Experimental Time line

\section{Thermal behavior testing - tail flick}

An IITC Tail Flick Apparatus (IITC Life Sciences, Inc.) was used with intensity set to 10 and beam strength set to 80 prior to starting the tail flick test. The rats' tails were darkened with a black marker to normalize the background of and response from the tail. Animals were restrained and placed upon the IITC Tail Flick Apparatus (IITC Life 
Sciences, Inc.) with their tails placed through a hole on the apparatus making it accessible to the light beam. The light beam was directed onto the proximal third of the tail of the animal where the incision and/or injection were located. The test was complete when the animal moved its tail away from the light beam or when the humane cut-off point of 10seconds was reached. Latencies to response were recorded. Animals were tested 3 times per time point.

\section{Mechanical behavior test - von frey}

Rats were placed upon a platform with the tail incision/injection area exposed for mechanical probing using the up-down (quantal) method. ${ }^{12}$ Four groups were analyzed: control, incision, incision with DON injection, and DON injection only. Using a $300 \mathrm{~g}(6.65 \mathrm{~N})$ Von Frey monofilament, pressure was applied in a downward perpendicular motion with the tip of monofilament onto the lateral margin of the incision, until the monofilament flexed. A positive response was noted when the animal moved its tail and/or vocalized. Animals were tested 3 times per time point.

\section{Immunohistochemistry}

Animals were deeply anesthetized with tribromoethanol $(2.5 \%$ w/v) (Sigma-Aldrich) and xylazine $(100 / \mathrm{ml})$ (Lloyd Laboratories) and perfused through the ascending aorta with $75 \mathrm{~mL}$ calcium-free Tyrode's solution, $\mathrm{pH} 7.3$, followed by $325 \mathrm{~mL}$ paraformaldehyde/ picric acid fixative. ${ }^{13}$ Tail skin tissues were collected placed in postfixative for fourhours at room temperature, and transferred to $10 \%$

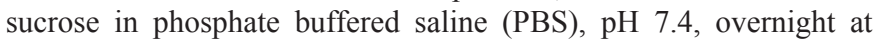
$4^{\circ} \mathrm{C}$. Tail skin tissue sections were cut at $20 \mu \mathrm{m}$ with a cryostat (Leica Microsystems) and mounted onto gel coated ProbeOn ${ }^{\mathrm{TM}}$ (Fisher Scientific) slides. The tissues were rinsed inside slide mailers (5

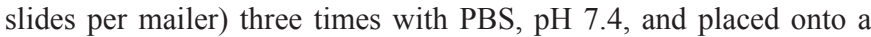
rocker for 10minutes after each wash. The primary antisera used were mouse anti-glutamate (1:40,000; Madl, Colorado State Univ.), rabbit anti-GLS (1:5,000; Curthoys, Colorado State Univ. $)^{14}$ and rabbit antiVGluT2 (1:2,000; Sigma-Aldrich). The anti-sera were placed into a blocking agent consisting of $0.5 \%$ polyvinylpyrollidone (PVP), $0.5 \%$ bovine serum albumin (BSA) in PBS with $0.3 \%$ Triton $\mathrm{X}-100$ (PBST), pH 7.4 and placed on a rocker in a cold room for 96hours. After the 96-hour incubation, the tissues were rinsed three times for 10 minutes each with PBS, $\mathrm{pH} 7.4$, and placed on a rocker during each rinse. Tissues were incubated in AlexaFluor $488 \AA$ conjugated goat anti-rabbit anti-sera $(1: 2000$; Invitrogen) or Alexafluor $555 \AA$ conjugated goat anti-mouse anti-sera (1:1500 Invitrogen) in (PBS-T) and incubated on a rocker for 60minutes at room temperature. Antisera were decanted and the tissues were washed three times for 10minutes per wash with PBS pH 7.4 and placed on a rocker between washes. ProLong Gold ${ }^{\mathrm{TM}}$ (Invitrogen) mounting medium was added to each section of tissue and coverslips applied. Slides were stored at room temperature for 24 hours, in a dark area, to allow for complete polymerization of mounting medium.

\section{Absorption controls}

Primary antiserum absorption control and secondary antiserum control experiments were performed for the GLS and VGLUT2 primary antisera and secondary antisera, respectively. For primary antiserum absorption controls, each diluted antiserum was incubated for 24 hours at $4^{\circ} \mathrm{C}$ with the respective antigen at $20 \mathrm{mg} / \mathrm{mL}$. Processing of DRG sections for immunofluorescence was carried out as mentioned above. Additional sections were incubated for fourdays at $4^{\circ} \mathrm{C}$ in either absorbed diluted antisera or non-absorbed diluted antisera. The secondary antisera control sections were incubated in PBS-T for fourdays at $4^{\circ} \mathrm{C}$ before continuing with routine processing (Figure 2).
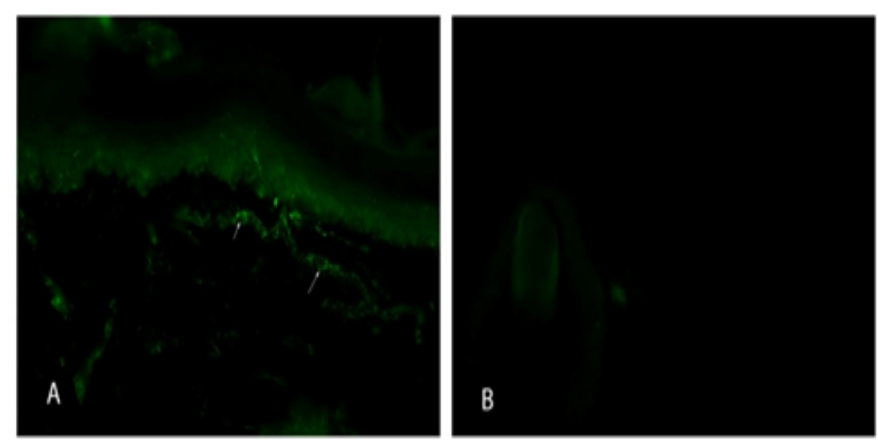

Figure 2 Absorption Control Rat Skin.

A. Cryostat section of rat skin stained with rabbit anti-GLS.

B. Staining (arrows) is abolished if antibody rabbit anti-GLS is first preabsorbed.

\section{Imaging}

All images were acquired using an Olympus BX51 epifluorescence microscope (Olympus; Center Valley, PA, USA), equipped with a SPOT RT740 quantitative camera (Diagnostic Instruments; Sterling Heights, MI, USA). VGluT2 images were acquired using a $40 \mathrm{X}$ objective. To ensure proper quantitation, all images were captured using a 1200 millisecond exposure with the gain set at one. Captured images were $1600 \times 1200$ pixels with 5.38 pixels per micrometer. There were a total of 1275 data points analyzed. Images were taken along the stratum lucidum border. ImageJ $(\mathrm{NIH})$ was used for analysis using the grid plug-in for standard sizing and the rectangular selection tool for taking random images along the stratum lucidum border. Sample size of the rectangular selection tool was 23408 pixels.

GLS images were acquired using a $60 \mathrm{X}$ objective. To ensure proper quantitation, all images were captured using a 2000-millisecond exposure with the gain set at 1 . Captured images were 16001200 pixels with 8.07 pixels per micrometer. There were a total of 218 nerve fibers evaluated. Images were taken within the dermal layer. ImageJ (NIH) was used for analysis, utilizing the free form drawing feature to outline nerve fibers and analyze their intensity.

GLU images were acquired using a $60 \mathrm{X}$ objective. To ensure proper quantitation, all images were captured using a 2000-millisecond exposure with the gain set at 1 . Captured images were $1600 \times 1200$ pixels with 8.07 pixels per micrometer. Three slides were used for the GLU anti-sera. There were a total of 106 nerve fibers evaluated. Images were taken within the epidermal layer. ImageJ (NIH) was used for analysis, utilizing the free form drawing feature to outline nerve fibers and analyze their intensity.

\section{Statistics}

Data from the analyses are reported as percentage change. A student's t-test, ANOVA or Fisher's exact test were used to determine differences between experimental and control groups (Prism version 6.0, GraphPad Software Inc., La Jolla, CA.). In all analyses, p-values less than 0.05 were considered significant. 


\section{Results}

\section{Behavior testing - mechanical}

The control and DON injection-only groups had no response to the $300 \mathrm{~g}(6.65 \mathrm{~N})$ monofilament. Within the incision only group, 12 out of 15 animals responded with tail movement or vocalization to the $300 \mathrm{~g}$ monofilament. Six of the 12 incision only animals that responded to mechanical stimulation received an infiltration/injection of $2 \mathrm{mM}$ DON into the incision site as described previously. The DON administration occurred 16 hours post-incision and animals were tested 24 and 48hours later. Following DON administration, no response was noted for five out of six animals to the $300 \mathrm{~g}$ monofilament (Figure $3)$. All untreated incision only animals $(n=6)$ responded to mechanical stimulation at 24 and 48 hours. No gender differences were noted.

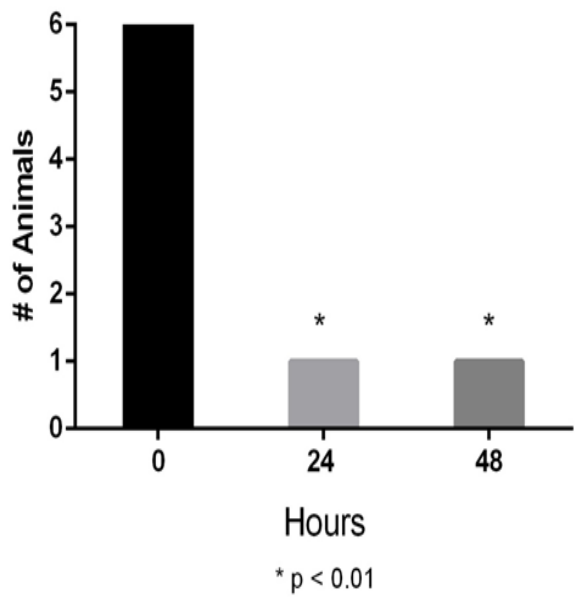

Figure 3 Mechanical Allodynia Post-incision.

Animals were tested for allodynic response using a $300 \mathrm{~g}(6.65 \mathrm{~N})$ Von Frey monofilament. Pressure was applied in a downward motion with the tip of monofilament perpendicular onto the lateral margin of the incision, until the monofilament flexed. A positive response was noted when the animal moved its tail and/or vocalized. Animals were tested 3times per time point. Before treatment all post-incisional animals responded $(n=6)$. At 24 and 48 hours post DON treatment, one out of six animals responded. Fishers exact test was used to determine significance.

\section{Behavior testing - thermal}

The control and DON injection only groups had no response to the thermal testing. Out of the animals that received an incision $(n=15)$, only five animals responded to thermal stimuli. The responders were given an injection/infiltration of $2 \mathrm{mM}$ DON into the incision site 16 hours post incision. These animals were tested 24 and 48 hours later and no animal showed an analgesic response. No gender differences were noted.

Immunoreactivity changes in the glutamateric biomarkers VGIuT2, GLS and GLU after the application of the GLS inhibitor DON

To examine the effect of DON on glutamate (GLU) we infiltrated the incision area with twenty $0.05 \mathrm{ml}$ aliquots of $2 \mathrm{mM}$ DON 16-hour post-surgical incision.

For GLS, ir was examined in the dermis (Figure 4). A total of 218 nerve fiber bundles were analyzed. The mean grayscale intensity (MGI) for control was 100.51 11.76 ru, for DON injection only $101.42 \pm 10.46 \mathrm{ru}$, incision only $153.43 \pm 3.24 \mathrm{ru}$, incision with DON 138.23 \pm 3.74 ru (Figure 5). A significant decrease in immunofluorescence was observed in the incision with DON group compared to incision only group. Our analysis found that GLS-ir decreased by approximately $27.9 \%$ in comparison to the incision only group after the administration of DON.
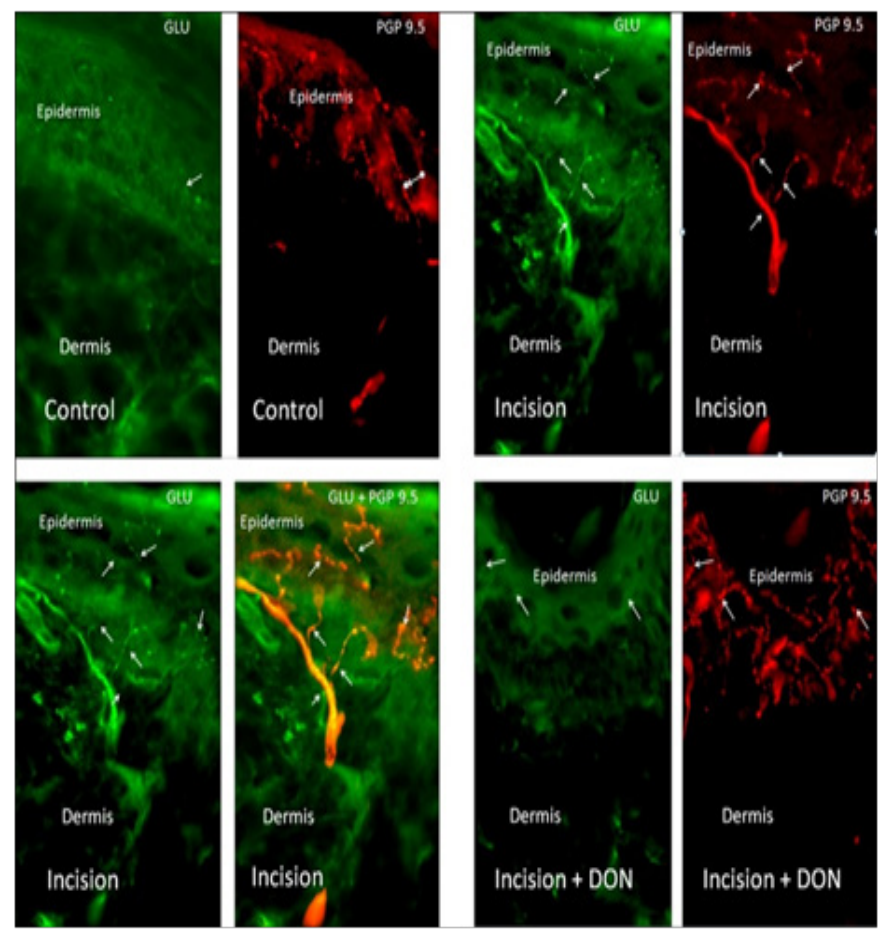

Figure 4 DON effect on immunoreactivity (ir) of VGluT2, GLS and GLU. White arrows point to areas of ir.
A) Control GLU
B) Control PGP 9.5
C) GLU Incision
D) PGP 9.5 Incision
E) GLU post incision
F) GLU + PGP 9.5 post incision
G) GLU post incision + DON
H) GLS I. GLU + PGP $9.5+$ DON post incision.

Note DON was administered I 6hours post incision.All images were acquired 64hours post-incision.

For GLU ir was measured in the epidermis (Figure 4). A total of 106 nerve fibers were analyzed. The MGI for control was $133.62 \pm 8.00 \mathrm{ru}$, for DON injection only $155.78 \pm 5.03 \mathrm{ru}$, incision only $180.61 \pm 3.47 \mathrm{ru}$, incision with DON: 139.09 \pm 6.02 ru (Figure 6). A significant decrease in immunofluorescence was observed in the incision with DON group compared to incision only group. Our analysis found that GLU-ir decreased by approximately $23 \%$ after the administration of DON.

For VGluT2, ir was measured in stratum lucidum of the epidermis (Figure 7). A total of 1275 areas were analyzed. The MGI for control was $101.31 \pm 31.48 \mathrm{ru}(\mathrm{n}=319)$, DON injection only $102.08 \pm 2.71 \mathrm{ru}$ $(\mathrm{n}=318)$, incision only $120.77 \pm 3.27 \mathrm{ru} \quad(\mathrm{n}=319)$, incision with DON $114.20 \pm 2.71 \mathrm{ru}(\mathrm{n}=319)$ (Figure 7). A significant decrease in 
immunofluorescence was observed in the incision with DON group compared to incision only group. Our analysis found that VGluT2-ir decreased by approximately $5.4 \%$ after the administration of DON.

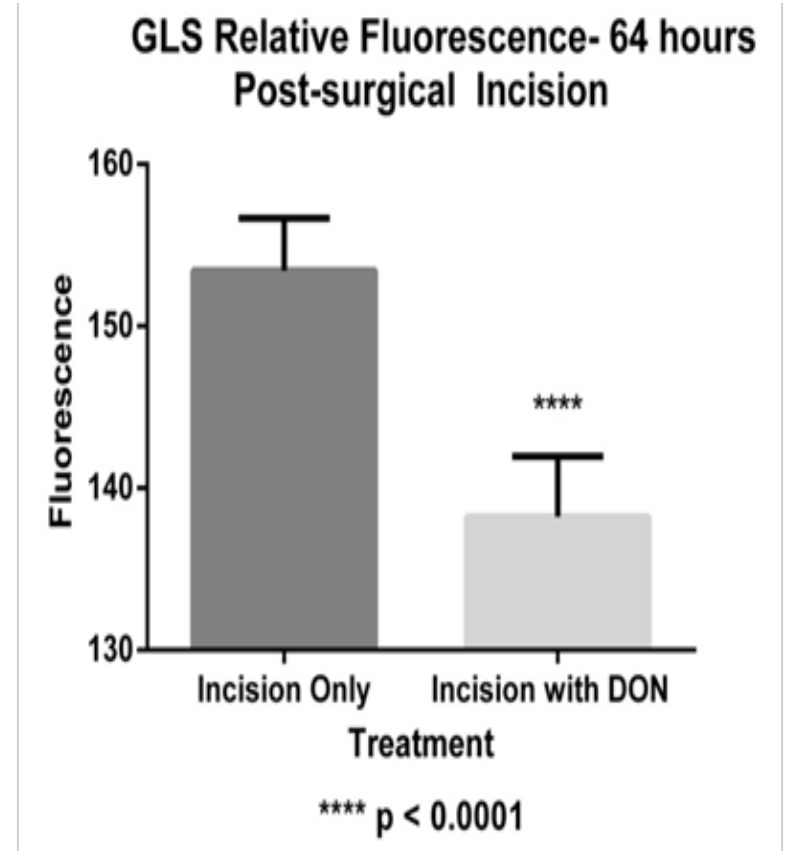

Figure 5 GLS Relative Immunofluorescence 64 hours Post-surgical Incision. Incision only $(n=109)$ 153.43 \pm 3.24 ; DON with incision $(n=77)$ 138.23 \pm 3.74 . A significant decrease in GLS immunofluorescence was observed in the incision with DON group compared to incision only group. $\mathrm{N}=$ number of nerve fiber bundles analyzed. Data reported as mean \pm sem.

\section{GLU Relative Fluorescence- 64 hours Post-surgical Incision}

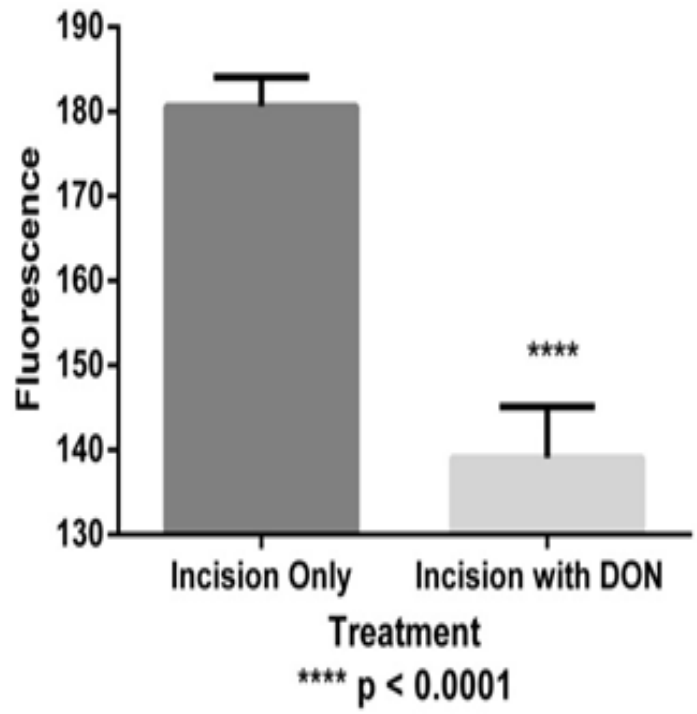

Figure 6 GLU Relative Immunofluorescence 64hours Post-surgical Incision. Incision only $(n=35) 180.61 \pm 3.47$; DON with incision $(n=34)$ 139.09 \pm 6.02 . A significant decrease in GLU immunofluorescence was observed in the incision with $\mathrm{DON}$ group compared to incision only group. $\mathrm{N}=$ number of nerve fibers analyzed. Data reported as mean \pm sem.

\section{VGluT2 Relative Fluorescence- 64 hours Post-surgical Incision}

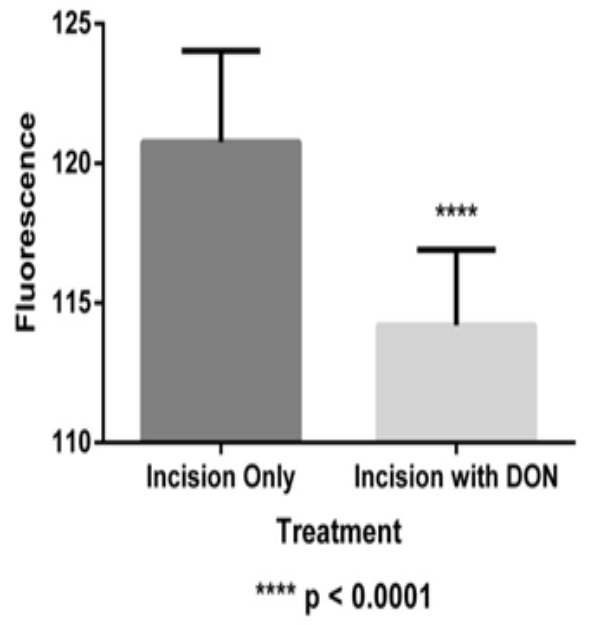

Figure 7VGluT2 Relative Immunofluorescence 64hours Post-surgical Incision with incision only and incision with DON injection.

Incision only $(n=3 / 9) \quad 120.77 \pm 3.27$; DON with incision $(n=3 / 9)|| 4.20 \pm 2.7 \mid$ A significant decrease in VGluT2 immunofluorescence was observed in the incision with DON group compared to incision only group. $\mathrm{N}=$ number of areas analyzed. Data reported as mean \pm sem.

\section{Discussion}

Current pharmacotherapy for the treatment of pain consists of topical analgesics, local anesthetics, opioids, NSAIDs, anti-epileptics, and anti-depressants. Although numerous agents exist for the treatment of pain, none have proven a panacea. Furthermore, none of these drugs act directly on the peripheral glutamatergic system, even though glutamate is a strong stimulator of primary afferent neurons. ${ }^{15,16}$ Since glutaminase is responsible for the neuronal production of glutamate from glutamine in peripheral afferent terminals, it is a potential therapeutic target for alleviating pain..$^{7,10,11}$ The GLS enzyme contains several regulatory sites, ${ }^{17-19}$ and several classes of glutaminase inhibitors, acting at both allosteric and enzymatic sites are effective analgesics when injected into the inflamed limb of adjuvant-induced arthritis (AIA) animals. ${ }^{6,7}$

One particular glutamine antagonist, 6-diazo-5-oxo-l-norleucine (DON), inhibits the enzyme, glutaminase. ${ }^{15,20}$ This compound was previously tested as a chemotherapeutic drug over 50years ago, ${ }^{21}$ however, toxicity limited its approval and use in humans. ${ }^{22}$ Originally discovered from a Streptomyces strain, DON was found to have tumor inhibitory action and was introduced into human cancer clinical trials. ${ }^{21,23}$ Its main chemotherapeutic effect is blockade of purine biosynthesis by inhibition of amido phosphoribosyl transferase (EC 2.4.2.14) and phosphoribosyl formyl glycinamidine synthase (EC 6.3.5.3), although it can block a number of amido transferases. ${ }^{24}$ DON has been used in at least 13 clinical trials, representing over 700 cancer patients. ${ }^{21,25,26}$

There have been anecdotal reports of pain relief following treatment with DON in cancer patients. A woman who received $480 \mathrm{mg} / \mathrm{m}^{2}$ "reported marked decrease in pain from a destructive lesion in the lumbar spine due to a fibrosarcoma." This pain relief lasted six weeks 
despite progression of the disease. ${ }^{26}$ Another report of analgesia was a fourteen-year old male with a vertebral tumor who reported a decrease in pain after $450 \mathrm{mg} / \mathrm{m}^{2}$ dose. ${ }^{25}$ These two examples of pain relief both involved tumor invasion of the spinal vertebrae. This is a highly painful condition and adequate pain relief is difficult to provide. ${ }^{27,28}$ DON inhibition of GLS activity in peripheral nerve terminals may have been part of the reason for pain relief in these patients.

\section{DON mechanism of action}

As mentioned previously, the GLS enzyme contains several regulatory sites. ${ }^{14-17,29-31}$ Several GLS inhibitors acting at both allosteric and enzymatic sites have been shown to be robust analgesics when injected into the inflamed limb of AIA animals, and the most potent analgesic was DON. ${ }^{7}$ The mechanism of action for DON is transport into nerve terminals via glutamine transporters ${ }^{32}$ and irreversible binding to the glutamine-binding site of GLS in the peripheral terminals. ${ }^{12}$ Replenishment of inhibited GLS in peripheral terminals takes severaldays as neuronal cell bodies (e.g. DRG) must transcribe, translate, and transport the newly synthesized GLS to the peripheral terminals, which can reside up to one meter away in humans. Because this process takes one to twodays, DON inhibition of GLS in peripheral terminals provides long-lasting analgesia as seen in these animal experiments. The qualities of having a long duration of action and analgesia as compared to anesthesia make DON a unique therapeutic agent in the local treatment of pain

A surgical incision is an acute painful injury. Following incision, wound inflammation causes an increase in the production of nociceptive molecules that augment both central and peripheral sensitization. ${ }^{33}$ These primary sensory neurons are glutamatergic, releasing glutamate from peripheral and central terminals via VGluT2 synaptic vesicles. ${ }^{34}$ Previous studies have demonstrated that glutamatergic metabolism is elevated in DRG neurons post-surgical incision and during adjuvantinduced arthritis (AIA), leading to hyperalgesia and allodynia. ${ }^{7,8}$ Our investigation was to evaluate whether DON, when injected into a surgical incision, would have a similar analgesic effect as observed in adjuvant-induced arthritis (AIA) animals. ${ }^{7}$

\section{Alterations in GLS,VGluT2, and GLU Immunoreactivity in the skin post-surgical incision}

Previous work in the aseptic incision model has shown a cytoplasmic elevation of GLS in affected DRG occurring betweendays 1 and 2, post-incision. ${ }^{8}$ However, the question remained as to whether this increased GLS is transported to peripheral terminals to allow for increased production of GLU. Upon examination of the skin 64hours post-surgical incision, we found that skin GLS-ir was elevated, supporting the narrative that elevated DRG GLS is transported to peripheral terminals in the aseptic incision model in the rat. ${ }^{7,8}$ The next question was whether this increased level of GLS allowed for increased production in GLU. To address this question, we examined GLU and its vesicular transporter, VGluT2, expression in the skin. Previous research ${ }^{8}$ has shown that VGluT2 expression is elevated in the DRG and that this increase in both VGLuT2 and GLS may mean that there is increased synthesis and release of glutamate from the peripheral terminals. The question still remained whether this increase in DRG VGluT2 expression was transported to the peripheral terminals in the aseptic incision model. Our findings revealed that VGluT2-ir is elevated in the skin post-surgical incision indicating that the increased expression of VGluT2 synthesized in the cell body is transported to the peripheral terminals in response to an incisional injury. Finally, GLU-ir was measured in the epidermis and our findings revealed that GLU-ir was elevated post-surgical incision. This expression profile is congruent with the role primary afferent neurons play in nociception, in both acute and chronic painful conditions. ${ }^{35,36}$ The final question was whether the application of the GLS inhibitor, DON, would decrease the levels of GLS-ir, VGluT2-ir, and GLU-ir. We conjectured that the irreversible binding of DON to GLS would result in protesomal degradation, thereby leading to decreased levels of GLS immunoreactivity, followed by a subsequent decrease in GLU- and VGluT2-ir. Our analysis found that GLS-ir decreased by approximately $27.9 \%$, VGluT2-ir by approximately $5.4 \%$, and GLUir by approximately $23 \%$ after the administration of DON. Other researchers have shown similar results at other nervous system sites. For example, Conti et al., showed when 0.25-1 mM DON was either injected intraparenchymally or applied epipially to the sensorimotor cortex of adult Sprague-Dawley rats,that GLU-ir was abolished in neuron perikarya. ${ }^{20}$ Bradford et al. ${ }^{37}$ showed both a decrease in GLU pool size and release from cerebrocortical synaptosomes when treated with DON $(5 \mathrm{mM}) .{ }^{37}$ When applied intrathecally to lumbar DRG's in rats, DON decreases GLS ir in DRG neuronal cell bodies withinhours of application, indicating cellular degradation of the inhibited enzyme. ${ }^{38}$ In summary, our analysis found that GLS-ir decreased by approximately $10.5 \%$, VGluT2-ir by approximately $6.5 \%$, and GLUir by approximately $23 \%$ after the administration of DON. Taken together, these results provide in vivo support for DON's mechanism of action. The GLU immunoreactivity results offer the most direct and compelling support for DON's mechanism of action. We would expect that an inhibitor of GLS to show decreased GLU immunoreactivity as GLU is the product of that enzyme and by inhibiting this enzyme less GLU would be produced.

\section{The behavioral effects of don on mechanical and thermal sensitivity post-surgical incision}

Previous studies have examined if inhibition of GLS in peripheral terminals would reduce elevated glutamate levels and provide pain relief during chronic inflammation. ${ }^{6}$ These studies have shown that following a single intra-plantar injection of DON, 3days post-AIA, thermal and mechanical responses were comparable to control animals and glutamate-ir appeared similar to controls. ${ }^{7}$ Another study showed that pre-treatment of rat hind paws with DON, along with co-administration of DON at the start of inflammation, decreased paw swelling by $10 \%{ }^{10}$ and reduced the number of activated neurons in the spinal cord as demonstrated by c-fos-ir.

To test the analgesic efficacy of DON in the post-surgical incision model, mechanical and thermal latencies were analyzed. When mechanical latency was tested, the control and DON injection-only groups had no response to the $300 \mathrm{~g}(6.65 \mathrm{~N})$ Von Frey monofilament. In the animals that received an incision $(\mathrm{n}=15), 12$ out of 15 responded to $300 \mathrm{~g}$ monofilament. Of the 12 animals that responded, six received a $1 \mathrm{ml}$ injection of $2 \mathrm{mM}$ DON 16 hours post incision and were tested 24 and 48hours after the injection. Of those tested, five out of six animals showed a significant analgesic response to the $2 \mathrm{mM}$ dose, indicating analgesic efficacy.

There was no significant decrease in thermal latency between the control and incisional animals. This finding is consistent with previous work in the tail incisional model. Weber et al. ${ }^{39}$ showed no significant difference in thermal response in their surgical incision model. However, the Brennan incisional hind paw and gastrocenimus models do show thermal sensitivity. ${ }^{40}$ These differences might well be 
determined by the variations in tissue composition between the tail, paw, and gastrocenimus.

\section{Conclusion}

In this study, we addressed the following hypothesis: Since glutaminase and VGluT2 are elevated in affected dorsal root ganglia neuronal soma post-surgical incision, then the application of a glutaminase inhibitor will result in a decrease in glutamate biomarkers in skin and provide analgesia. The results of these studies demonstrate that skin immunoreactivity of GLS, GLU and VGluT2, in this surgical incision model, increase after post-surgical incision. This elevation in immunoreactivity is attenuated by the application of the glutaminase inhibitor, DON. The decrease in these biomarkers is consistent with the mechanism of action proposed for DON. Furthermore, the application of DON post-surgical incision produced analgesia by attenuating allodynia and producing a mechanical response similar to control animals. However, the results of thermal sensitivity were not significant, similar to other reports. ${ }^{39,40}$ Overall, our results corroborate previous findings using DON in an animal inflammatory model. In summary, these data offer an exciting insight into a novel treatment of pain though the inhibition of the enzyme that produces the principle neurotransmitter of primary afferent neurons, i.e., glutamate. This approach lays a preclinical foundation for DON and provides additional support for further investigation into this novel treatment of pain.

\section{Acknowledgements}

None.

\section{Conflict of interest}

Author declares that there is no conflict of interest.

\section{References}

1. Johansen A, Romundstad L, Neilsen CS, et al. Persistent postsurgical pain in a general population: Prevalence and predictors in the Tromsø study. Pain. 2012;153(7):1390-1396.

2. Badar P, Echtle D, Fonteyne V, et al. Post-operative pain management: Guidelines on pain management. European Society of Urology. 2010;4:61-82

3. Kissin I. Preemptive Analgesia: Problems with assessment of clinical significance. Methods Mol Biol. 2010;617(4):475-482.

4. Tao YX, Gu J, Stephens RL. Role of spinal cord glutamate transporter during normal sensory transmission and pathological pain states. Molecular Pain. 2005;1:30.

5. Siegel GJ. Basic neurochemistry: Molecular, cellular, and medical aspects. Philadelphia: Lippincott Williams \& Wilkins; 1999.

6. Miller KE, Balbas JC, Benton RL, et al. Glutaminase immunoreactivity and enzyme activity is increased in the rat dorsal root ganglion following peripheral inflammation. Pain Res Treat. 2012; 2012:414697.

7. Miller KE, Hoffman EM, Sutharshan M, et al. Glutamate pharmacology and metabolism in peripheral primary afferents: Physiological and pathophysiological mechanisms. Pharmacology and Therapeutics. 2011;130(2):283-309.

8. Crosby HA, Ihnat M, Spencer D, et al. Expression of glutaminase and vesicular glutamate transporter type 2 immunoreactivity in sacral dorsa root ganglia following a surgical tail incision. Pharmacy and Pharmacology International Journal. 2015;2(3):23-31.
9. Zhang Z, Wang T, Miller KE, Accumulation of glutaminase, VGluT2, and substance $\mathrm{P}$ in sciatic nerve following adjuvant-induced arthritis in rat. Society for Neuroscience, 586.10.

10. Hoffman EM, Miller KE. Peripheral inhibition of glutaminase reduces carrageenan-induced Fos expression in the superficial dorsal horn of the rat. Neurosci Letters. 2010;472(3):157-160.

11. Hoffman EM, Edwards KM, Herzog BH, et al. Intrathecal glutaminase inhibition with 6-diazo-5-oxo-L-norleucine in rat dorsal root ganglion neurons. Proceedings of the Society for Neuroscience 737.5. 2006.

12. Shapiro RA, Clark VM, Curthoys NP. Inactivation of rat renal phosphate-dependent glutaminase with 6-diazo-5-oxo-L-norleucine. Evidence for interaction at the glutamine binding site. $J$ Biol Chem. 1979;254(8):2835-2838.

13. Kvamme E, Torgner I. Regulatory effects of fatty acyl-coenyme A derivatives on phosphate-activated pig brain and kidney glutaminase in vitro. Biochem J. 1975;149(1):83-91.

14. Guidelines for the care and use of animals in neuroscience. Washington D.C: The National Academies Press; 2003:1-223.

15. Dixon WJ. Efficient analysis of experimental observations. Annual Rev Pharmacol Toxicol. 1980;20(1):441-462.

16. Hoffman EM, Schechter R, Miller KE. Fixative composition alters distributions of immunoreactivity for glutaminase and two markers of nociceptive neurons, Nav1.8 and TRPV1, in the rat dorsal root ganglion. $J$ Histochem Cytochem. 2010;58(4):329-344.

17. Curthoys, NP, Watford, M. Regulation of glutaminase activity and glutamine metabolism. Annu Rev Nutr. 1995;15(1):133-159.

18. Davidson EM, Coggeshall RE, Carlton SM. Peripheral NMDA and non-NMDA glutamate receptors contribute to nociceptive behaviors in the rat formalin test. Neuroreport. 1997;8(4):941-946.

19. Lawand NB, Willis WD, Westlund KN. Excitatory amino acid receptor involvement in peripheral nociceptive transmission in rats. European Journal of Pharmacology. 1997;324(2-3):169-177.

20. Conti F, Minelli A. Glutamate immunoreactivity in rat cerebral cortex is reversibly abolished by 6-diazo-5-oxo-L-norleucine. J Histochem Cytochem. 1994;42(6):717-726.

21. Magill GB, Myers WP, Reilly HC, et al. Pharmacological and initial therapeutic oberservations on 6-diazo-5-oxo-L-norleucine (DON) in human neoplastic disease. Cancer. 1957;10(6):1138-1150.

22. Ovejera AA, Houchens DP, Cantane R. Efficacy of 6-diazo-5-oxo-1-norleucine and N-6-diazo-5-oxo-norleucine against experimental tumors in conventional and nude mice. Cancer Res. 1979;39(8):3220-3224.

23. Dion H, Fusari S, Jakubowski ZL, et al. 6-Diazo-5-oxo-L-norleucine, a new tumor-inhibitory substance. II. 1 Isolation and characterization. $J$ Am Chem Soc. 1956;78(13):3075-3077.

24. Ahluwalia G, Grem J, Hao Z, et al. Metabolism and action of amino acid analog anti-cancer agents. Pharmacol Ther. 1990;46(2):243-271.

25. Lynch G, Kemeny, N, Casper E. Phase II evaluation of DON (6-Diazo-5-Oxo-L-Norleucine) in patients with advanced colorectal carcinoma. Am J Clin Oncol. 1982;5(5):541-543.

26. Kovach J, Eagan R, Rubin J, et al. Phase I and pharmacokinetic studies of DON. Cancer Treat Rep. 1981;65(11-12):1031-1036.

27. Mantyh PW, Aoki Y, Ohtori S. Mechanisms that generate and maintain bone cancer pain. Novartis Found Symp. 2004;260:221-240.

28. Takahashi K, Aoki Y, Ohtori S. Resolving discogenic pain. Eur Spine J 2008;17(Suppl 4):428-431. 
29. Kvamme E, Roberg B, Johansen L, et al. Interrelated effects of calcium and sulfhydryl reagents on renal phosphate-activated glutaminase. Contrib Nephrol. 1988;63:156-160.

30. Kvamme E, Torgner IA, Roberg B. Evidence indicating that pig renal phosphate-activated glutaminase has a functionally predominant external localization in the inner mitochodrial membrane. J Biol Chem. 1991;266(20):131185-131192.

31. Shapiro RA, Curthoys NP. Characterization of the apparent rates of glutamine transport in rat renal mitochondria. FEBS Letters. 1978;91(1):49-52.

32. Low SY, Taylor PM, Ahmed A, et al. Substrate-specificity of glutamine transporters in membrane vesicles from Rat Liver and skeletal muscle investigated using amino acid analogues. Biochem J. 1991;278(1):105-111.

33. Fang X, McMullan S, Lawson SN, et al. Electrophysiological differences between nociceptive and non-nociceptive dorsal root ganglion neurones in the rat in vivo. $J$ Physiol. 2005;565(Pt3):927-943.

34. Chambille I, Rampin O. AMPA glutamatergic receptor-immunoreactive subunits are expressed in lumbosacral neurons of the spinal cord and neurons of the dorsal root and pelvic ganglia controlling pelvic functions in the rat. Brain Res. 2002;933(1):66-80.
35. Scherrer G, Low SA, Wang X, et al. VGluT2 expression in primary afferent neurons is essential for normal acute pain and injury-induced heat hypersensitivity. Proc Natl Acad Sci US A. 2010;107(51):22296-22301.

36. Rogoz K, Lagerström MC, Dufour S, et al. VGluT2-dependent glutamatergic transmission in primary afferents is required for intact nociception in both acute and persistent pain modalities. Pain. 2012;153(7):1525-1536.

37. Bradford H, Ward H, Foley P. Glutaminase inhibition and the release of neurotransmitter glutamate from synaptosomes. Brain Res. 1989;476(1):29-34.

38. Ottersen OP, Langmoen IA, Gjerstad L. The glutamate synapse as a therapeutical target: Molecular organization and pathology of the glutamate synapse. Amsterdam: Elsevier; 1998;116:441-458.

39. Weber, J, Loram, L, Mitchell, B, et al. A model of incisional pain: The effects of dermal tail incision on pain behaviours of Sprague Dawley rats. J Neurosci Methods. 2005;145(1-2):167-173.

40. Brennan TJ, Vandermeulen EP, Gebhart G. Characterization of a rat model of incisional pain. Pain. 1996;64(3):493-502. 